\title{
Treatment of Non-Colorectal Liver Metastases
}

\author{
Chair: \\ Henning Wege ${ }^{a}$ Ansgar W. Lohse ${ }^{a}$ \\ Participants: Dirk Arnold ${ }^{b}$ Gerd Otto $^{c}$ Arndt Vogel ${ }^{d}$ \\ ${ }^{a}$ l. Department of Internal Medicine, University Medical Center Hamburg-Eppendorf, Hamburg, Germany, \\ ${ }^{b}$ Department of Medical Oncology, Tumor Biology Center, Freiburg i.Br., Germany, \\ ${ }^{\mathrm{C}}$ Department of Transplantation and Hepatobiliopancreatic Surgery, University Medical Center Johannes Gutenberg University, \\ Mainz, Germany, \\ ${ }^{\mathrm{d}}$ Department of Gastroenterology, Hepatology and Endocrinology, Hannover Medical School, Hanover, Germany
}

\section{Question 1: How crucial is a pre-treatment biopsy of non-colorectal cancer (CRC) liver metastases to detect changes in tumor biology in comparison to the primary tumor, in particular with regard to targeted therapy?}

Vogel: At the moment, we do not have any targeted therapy available that requires a re-biopsy of a histologically proven tumor. However, this will likely change in the future. One early example for this is the METIV trial in patients with hepatocellular carcinoma (NCT01755767). In this trial, only patients with tumors with high c-MET expression are randomized, and emerging data indicate that c-MET expression might increase during first-line treatment of hepatocellular carcinoma. Therefore, patients with low c-MET expression in their primary tumor at initial diagnosis may be re-biopsied to meet the inclusion criteria of the study.

Otto: In view of the genetic variability of metastatic disease, a biopsy to detect changes in tumor biology does not impact the decision for surgery in hepatic metastases of non-CRC primaries. It may, however, be important for decisions concerning systemic therapy, e.g. in breast cancer. Deeper molecular insights into organ specificity in metastatic disease may help to optimize therapy in the future. Nevertheless and so far, treatment decisions are usually adjusted to the histological profile and biomarkers identified in the primary tumor.

Arnold: Above all, the biopsy is sometimes essential to differentiate between the onset of metastases from the known disease and the potential development of a secondary malignancy. Notably, also metachronous liver-limited metastatic relapse may occur late. A biopsy with focus on the characterization of the tumor biology is an interesting approach, since we do know that heterogeneity between the primary tumor and liver metastases may exist, as well as a biologic variability between different biopsy sites even within the same liver metastasis. Additionally, not all molecular characteristics are consistent over time, although most markers are relatively 'stable', which means that they show a high concordance with the primary tumor. Others are not, and still many others are functionally irrelevant, although they bear molecular information. So, I am also not too 'pushy' to promote this approach outside of dedicated clinical trials.

And beyond that, I am sure that newer techniques, such as the analysis of circulating tumor DNA (extracted from blood samples), will very likely replace tumor biopsy because it allows easier access to the biomaterial and may offer a more accurate representation of the actual tumor biology, as we have learned from the experience in non-small cell lung cancer. For this entity, molecular diagnosis from cell-free DNA has recently become the standard to define the best targeted treatment.

\section{Question 2: Is it justified to adopt successful surgical strategies from the treatment of CRC liver metastases for non-CRC metastases or do we need confirmative trials?}

Otto: The adoption of surgical principles from the treatment of CRC metastases for non-CRC hepatic lesions is justified. 20 years ago, surgical results in CRC metastases were comparable to contemporary results in non-CRC metastases. Even if it remains unclear whether hepatic surgery helps to improve long-term survival in non-CRC hepatic metastases, some prognostic factors appear to be uniformly valid for CRC and non-CRC hepatic involvement:

\section{KARGER}

Fax +497614520714

\section{() 2015 S. Karger GmbH, Freiburg}

$1662-6664 / 15 / 0316-0441 \$ 39.50 / 0$
PD Dr. med. Henning Wege

I. Medizinische Klinik und Poliklinik

Universitätsklinikum Hamburg-Eppendorf

Martinistraße 52, 20246 Hamburg, Germany

h.wege@uke.de 
number and size of metastases and bilobar involvement are just marginally important for the prognosis as long as R0 resection is achieved, extrahepatic disease and a short interval between primary and secondary treatment are strong negative prognostic factors, repeated resections prolong survival. In general, overall survival is the same after each resection, and this is by far the strongest factor supporting the argument that surgery may be capable of prolonging survival in non-CRC hepatic metastases.

Arnold: We clearly need more trials in both the curatively intended approaches as well as in the palliative-ablative setting, especially to determine the quantity of the benefit, and to compare this to systemic treatment alone. Personally, I am not so much interested in a direct comparison of resection versus ablation, or a comparative analysis of different ablative methods. We know that surgical resection provides the only chance of a complete (100\%) tumor ablation. However, I wonder whether the relatively small differences in local tumor eradication rates between the different ablative methods and resection are clinically relevant with respect to the overall prognosis of metastasized patients. In this discussion, the consideration of invasiveness on the one hand and of potential treatment-related risks on the other hand is highly important.

Vogel: I think that the tumor biology, with respect to occurrence and localization of metastases, between CRC and other gastrointestinal cancers is too different to allow for an extrapolation of established CRC treatment strategies. Moreover, we do not even have enough prospective data to be sure about the best way to treat patients with more advanced CRC liver metastases. Therefore, the question remains: Who should be treated with chemotherapy at which time point, and who should be resected at all?

\section{Question 3: What are the possible advantages of resection or local ablative therapy for non-CRC liver metastasis instead of further systemic therapy in a metastasized malignant disease?}

Vogel: Multimodal therapies facilitate the cure of a subset of patients with metastatic disease and/or may at least increase their survival. Moreover, some patients will have the chance of chemotherapy-free time.

Arnold: I agree! After systemic control is provided by means of systemic treatment, resection/ablation will allow to discontinue systemic treatment after a relative short treatment period and to leave the patient on 'TWIST' (which stands for time without symptoms and treatment). This will likely impact on the quality of life and reduce the onset of cumulative toxicities. And this will very likely have an impact on the prognosis, also in a non-curative setting; i.e., this 'no evidence of disease' period will add to the overall prognosis. In other words, resection/ablation may also be seen as a 'treatment line' with its own progression-free survival interval contributing to overall survival. In addition, and equally important, time without systemic chemotherapy will avoid - or at least postpone - the development of resistance to the respective systemic treatment, which may then successfully be used or reused at a later point.

Otto: There is no evidence-based advantage of resection compared to local ablative therapy. In view of a few case-control studies, a survival advantage may be assumed. In my opinion, the strongest argument is the afore-mentioned survival advantage after repeated resection. As long as hepatic involvement can be eliminated (or reduced) and additional extrahepatic lesions are not evident, repeated resection prolongs survival. However, I also assume that these positive outcomes are at least partly influenced by a favorable tumor biology.

\section{Question 4: Are perioperative treatment strategies amenable to non-CRC liver metastases?}

Otto: In multicenter reviews, perioperative chemotherapy appeared to prolong survival in non-CRC liver metastases. However, facing the marginal results of the EORTC 40983 study (surgery vs. surgery plus perioperative chemotherapy) in CRC metastases, it would be surprising to detect an advantage of perioperative ('preemptive') versus 'therapeutic' chemotherapy for non-CRC liver metastases.

Vogel: At the moment, we do not know which patients with non-CRC liver metastasis really benefit from resection. In principle, perioperative treatment strategies should be amenable to nonCRC tumors, but they should be tested in prospective trails. Preoperative treatment might not only assist with bringing patients to surgery but may also help to identify patients that benefit the most from surgery (favorable tumor biology).

Arnold: This depends on the treatment goal and tumor entity, of course. In most of these situations, systemic treatment is the mainstay and standard of care, and patients should not be 'undertreated' with this approach. Beyond that, there are two more benefits of a successful systemic treatment: i) response to systemic treatment may shrink the tumor and allow a less invasive ablative strategy, or even enable local therapy at all, and ii) we know that response to systemic treatment is an excellent prognostic parameter in almost all malignant diseases, patients with a good response are those with favorable prognosis, and the good prognosis may justify ablative or invasive and potentially risky treatment approaches.

Question 5: For non-CRC liver metastases, is resection always the first choice compared to local ablative approaches in patients with normal liver function?

Arnold: Not at all! We should rather think of using all available approaches as a 'toolbox' of ablative strategies, including resection, all local ablative techniques, which are limited to isolated sites (e.g. 
radiofrequency ablation or novel radiation techniques, such as intensity-modulated radiation therapy), and even consider local embolization techniques as part of this 'toolbox'. The question which technique to use as first-line treatment should be discussed in a multidisciplinary team and be based on: i) localization and number of sites (technical considerations), ii) overall prognostic evaluations (how 'curative' is the situation), iii) patient conditions (what about comorbidity, toxicity, life expectancy), and iv) availability of the respective method at the treating institution. Resection is clearly the most 'curative' approach as it allows a local 100\% tumor control; however, in the context of a palliative setting, this may not necessarily be the best option in all patients.

Otto: In my opinion, it is also not justified to absolutely prefer resection over other forms of ablative techniques as long as the selected therapy is capable of achieving an R0 situation. Depending on the approach, size and number of lesions matter. Naturally, the decision and treatment selection should always be based on an interdisciplinary decision. In addition, for reaching this decision, tumor origin, histology, and the time course of the disease (synchronous vs. metachronous hepatic involvement) have to be considered. The poorer the prognoses, the more reluctant I would be to use resection for non-CRC metastases. There is some evidence to be less reluctant in genitourinary or breast cancer metastases as compared to gastrointestinal tumors, in particular gastric, esophageal, and pancreatic malignancies.

Vogel: Hepatocellular carcinoma is probably the tumor in which local therapies are most often used. So far, almost all comparative studies - albeit most of them are retrospective - suggest that resection is better than any other local treatment. Nevertheless, and as already mentioned, treatment decisions should be made in a multidisciplinary tumor conference by taking all tumor- and patient-related factors into account.

\section{Question 6: In your opinion, what is the most urgent trial to advance the therapy of non-CRC liver metastases?}

Arnold: There are so many interesting questions to be addressed, in particular regarding various ablative methods in different tumor types in diverse therapeutic situations. However, not a single trial is the most urgent, since we have to prospectively collect data to refine the 'toolbox' approach: i) which method should be used in which localization, and ii) what are the outcome parameters and what is the contribution of a local ablative method to these outcome parameters (progression-free survival, overall survival, quality of life, treatment costs).

Vogel: We need to know which patients really benefit from resection in terms of improved survival.

Otto: In order to elucidate the role of resection in non-CRC metastases, patients with resectable hepatic disease and without extrahepatic lesions should be included in a randomized study comparing resection alone to systemic treatment alone. Such a study would require a multi-institutional cooperation to accomplish recruitment of separate cohorts based on the histological entity.

\section{Participants}

Prof. Dr. Dirk Arnold

Klinik für Internistische Onkologie

Klinik für Tumorbiologie

Breisacher Straße 117, 79106 Freiburg, Germany

arnold@tumorbio.uni-freiburg.de

Prof. Dr. Gerd Otto

Klinik für Allgemein-, Viszeral- und Transplantationschirurgie

Johannes Gutenberg-Universität Mainz

Langenbeckstraße 1, 55131, Mainz, Germany

gerd.otto@unimedizin-mainz.de

Prof. Dr. Arndt Vogel

Klinik für Gastroenterologie, Hepatologie und Endokrinologie

Medizinische Hochschule Hannover

Carl-Neuberg-Straße 1, 30625 Hannover, Germany

vogel.arndt@mh-hannover.de 